\title{
Stereospecific Synthesis of a Carbene- Generating Angiotensin II Analogue for Comparative Photoaffinity Labeling: Improved Incorporation and Absence of Methionine- Selectivity
}

\author{
Dany L. Fillion, Maud Deraët, Brian J. Holleran and Emanuel Escher*
}

Department of Pharmacology, Faculty of Medicine, Université de Sherbrooke, Sherbrooke, Québec, Canada, J1H 5N4

* Corresponding author: Emanuel Escher, Ph.D., Department of Pharmacology, Faculty of Medicine, Université de Sherbrooke, 3001-12th Avenue North, Sherbrooke, Québec, Canada, J1H 5N4. Phone: (819) 564-5346. Fax: (819) 564-5400. E-mail: Emanuel.Escher@USherbrooke.ca

\section{Supporting Information}

\section{TABLE OF CONTENTS :}

Physicochemical characterization of compound Fmoc-12 S2

${ }^{1}$ H NMR spectra for compound Fmoc-12 S3

${ }^{13}$ C NMR spectra for compound Fmoc-12 S4

EI-HRMS spectra for compound Fmoc-12 S5

Physicochemical characterization of compound $\mathbf{1 3} \quad$ S6

$\begin{array}{ll}\text { RP-HPLC chromatogram for compound } \mathbf{1 3} & \text { S7 }\end{array}$

MALDI/TOF-MS spectra for compound $\mathbf{1 3} \quad$ S8 
$\underline{\text { Physicochemical characterization of compound Fmoc-12 }}$

\begin{tabular}{|c|c|}
\hline $\begin{array}{l}\text { Melting Point } \\
\qquad\left({ }^{\circ} \mathrm{C}\right)\end{array}$ & $129-130$ \\
\hline $\begin{array}{c}{[\alpha]_{\mathrm{D}}^{25}} \\
\left(\mathrm{c}=\mathbf{1 . 0 6}, \mathrm{CHCl}_{3}\right)\end{array}$ & +36.70 \\
\hline $\begin{array}{c}{ }^{1} \mathrm{H} \text { NMR } \\
\left(\mathrm{CDCl}_{3}, 350 \mathrm{MHz}, \delta(\mathbf{p p m})\right)\end{array}$ & $\begin{array}{l}\delta 7.78\left(\mathrm{~d}, \mathrm{~J}=7.6 \mathrm{~Hz}, 2 \mathrm{H}, H_{4,5}-\mathrm{Fmoc}\right), 7.55(\mathrm{~d}, \mathrm{~J}=7.1 \\
\left.\mathrm{Hz}, 2 \mathrm{H}, H_{1,8}-\mathrm{Fmoc}\right), 7.42\left(\mathrm{t}, \mathrm{J}=7.3 \mathrm{~Hz}, 2 \mathrm{H}, H_{3,6}-\mathrm{Fmoc}\right. \\
), 7.30\left(\mathrm{td}, \mathrm{J}=7.4,1.1 \mathrm{~Hz}, 2 \mathrm{H}, H_{2,7}-\mathrm{Fmoc}\right), 7.11(\mathrm{q}, \mathrm{J}= \\
8.3 \mathrm{~Hz}, 4 \mathrm{H}, m-H+o-H), 5.16(\mathrm{~d}, \mathrm{~J}=8.0 \mathrm{~Hz}, 1 \mathrm{H}, \mathrm{NH}), \\
4.68(\mathrm{q}, \mathrm{J}=7.5 \mathrm{~Hz}, 1 \mathrm{H}, \alpha-\mathrm{CH}), 4.51(\sim \mathrm{t}, \mathrm{J}=8.7 \mathrm{~Hz}, \\
1 \mathrm{H}, \mathrm{O}-\mathrm{CHH}-\mathrm{Fmoc}), 4.40(\sim \mathrm{t}, \mathrm{J}=8.7 \mathrm{~Hz}, 1 \mathrm{H}, \mathrm{O}-\mathrm{CH} H- \\
\text { Fmoc), } 4.20(\sim \mathrm{t}, \mathrm{J}=6.5 \mathrm{~Hz}, 1 \mathrm{H}, \mathrm{CH}-\mathrm{Fmoc}), 3.17(\mathrm{ddd}, \\
\left.\mathrm{J}=21.0,13.8,5.6 \mathrm{~Hz}, 2 \mathrm{H}, \beta-\mathrm{CH} H_{2}\right)\end{array}$ \\
\hline $\begin{array}{c}{ }^{13} \mathrm{C} \mathrm{NMR} \\
\left(\mathrm{CDCl}_{3}, 75 \mathrm{MHz}, \delta(\mathrm{ppm})\right)\end{array}$ & $\begin{array}{c}174.75,155.61,143.63,141.35,137.30,129.82,127.80 \\
127.07,126.64,124.93,120.04,66.93,54.22,47.11, \\
37.21,33.76,31.98\end{array}$ \\
\hline EI-HRMS & $\begin{array}{c}\mathrm{m} / \mathrm{z} \text { calculated for } \mathrm{C}_{26} \mathrm{H}_{20} \mathrm{~N}_{3} \mathrm{O}_{4} \mathrm{~F}_{3}[\mathrm{M}+\mathrm{H}]^{+}: 495.1406 \\
\text { m/z founded }[\mathrm{M}+\mathrm{H}]^{+}: 495.1397 \pm 0.0015\end{array}$ \\
\hline
\end{tabular}




\section{$\underline{{ }^{1} \text { H NMR spectra for compound Fmoc-12 }}$}

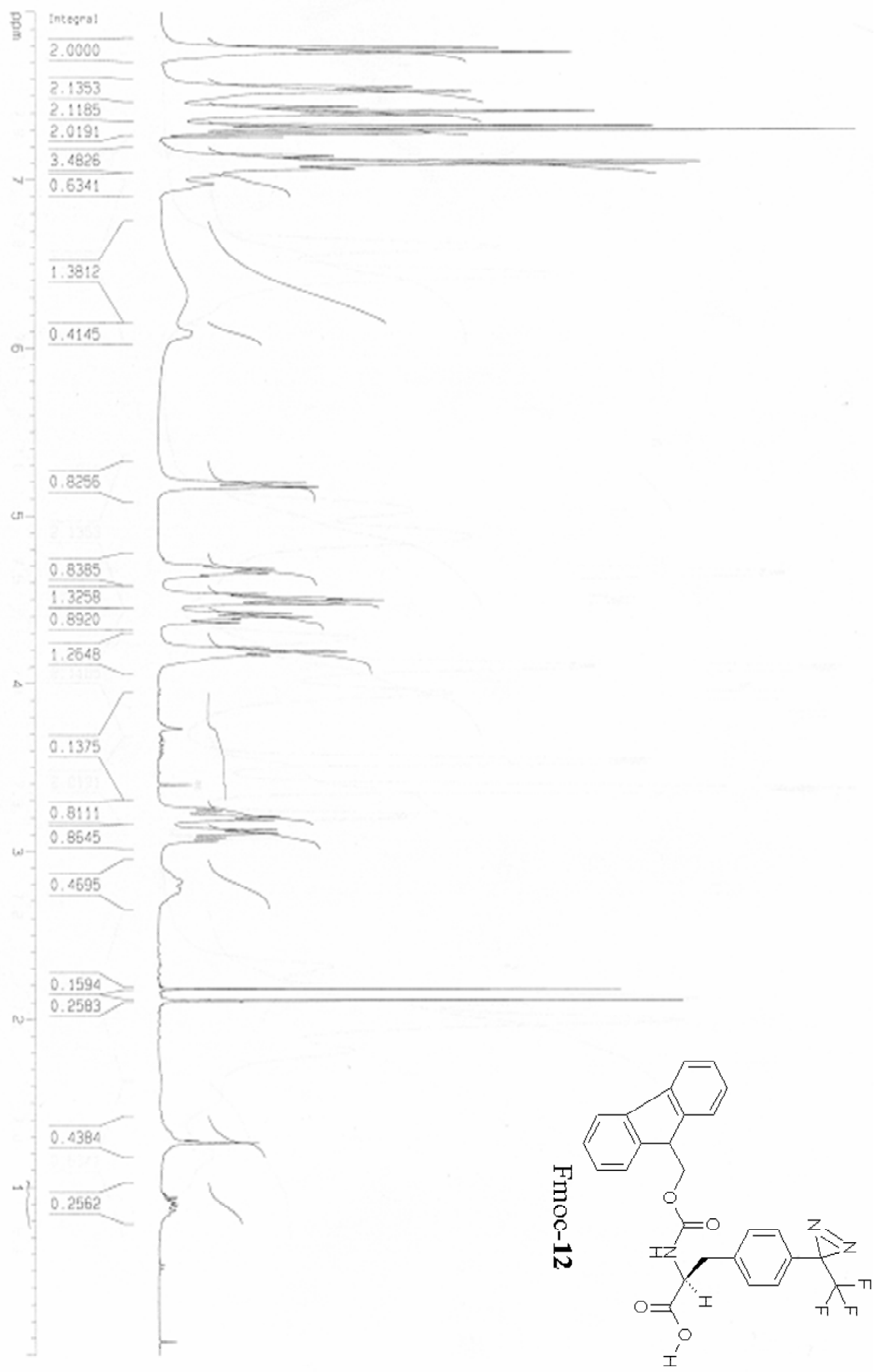


${ }^{13}$ C NMR spectra for compound Fmoc-12

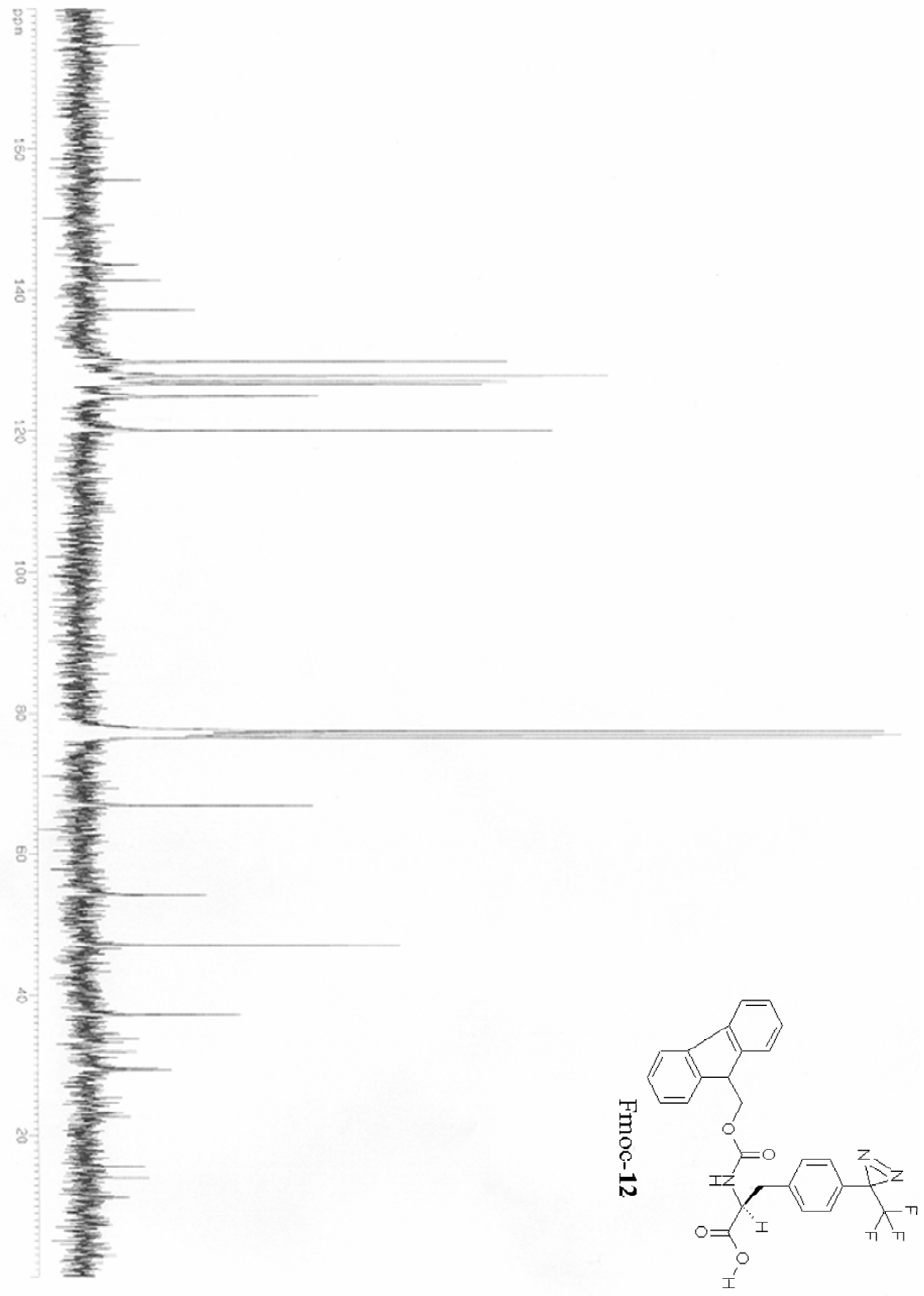


EI-HRMS spectra for compound Fmoc-12

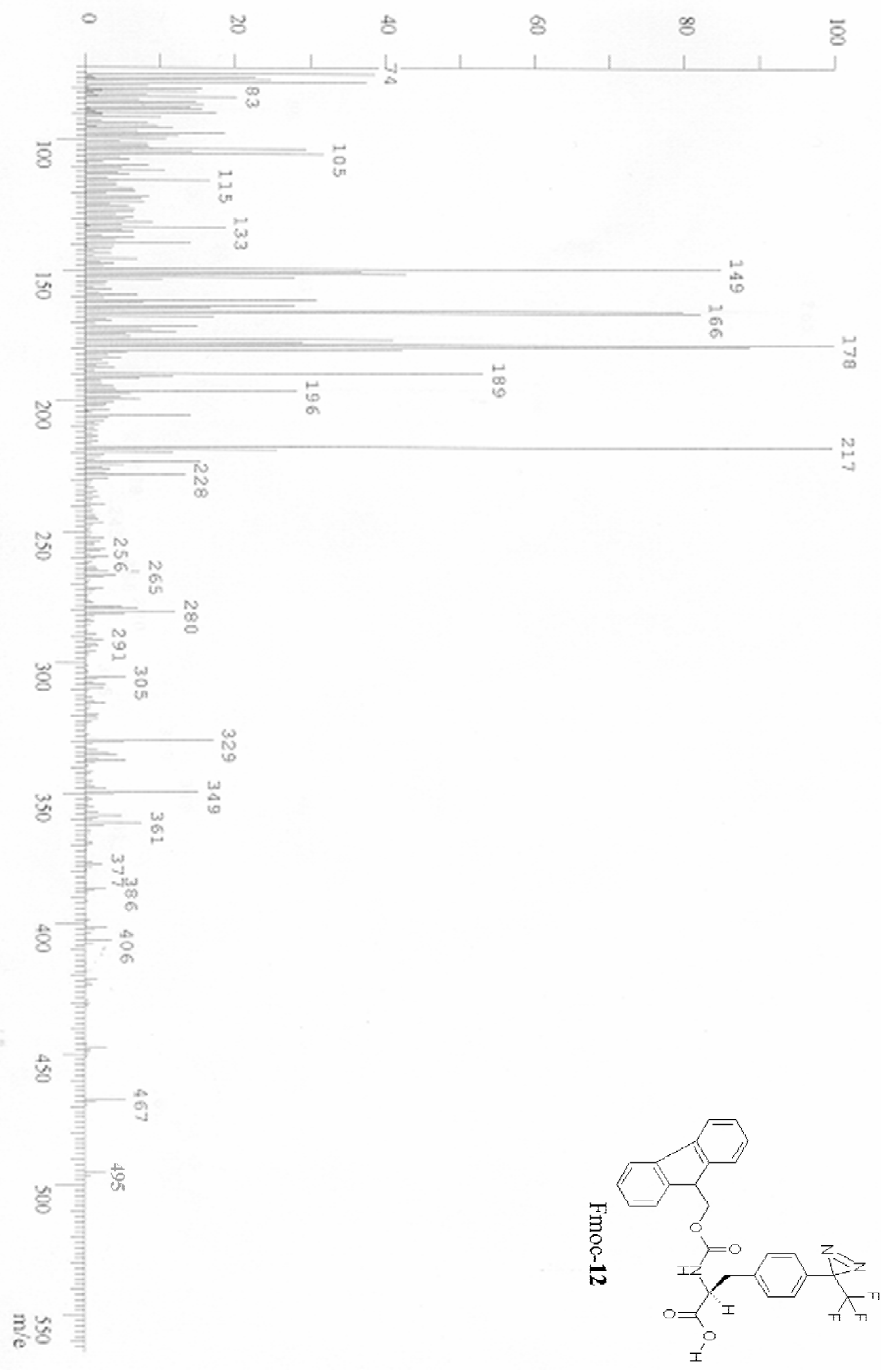


Physicochemical characterization of compound $\mathbf{1 3}$

\begin{tabular}{|c|c|}
\hline RP-HPLC & Single signal with a minimum of 95\% purity \\
\hline MALDI/TOF-LRMS & $\mathrm{m} / \mathrm{z}$ calculated for $\left[\operatorname{Sar}^{1}, \mathrm{Tdf}^{8}\right]$ AngII $[\mathrm{M}+\mathrm{H}]^{+}: 1097$ \\
& $\mathrm{~m} / \mathrm{z}$ founded $[\mathrm{M}+\mathrm{H}]^{+}: 495.1397 \pm 0.0015$ \\
\hline
\end{tabular}


$\underline{\text { RP-HPLC chromatogram for compound } \mathbf{1 3}}$

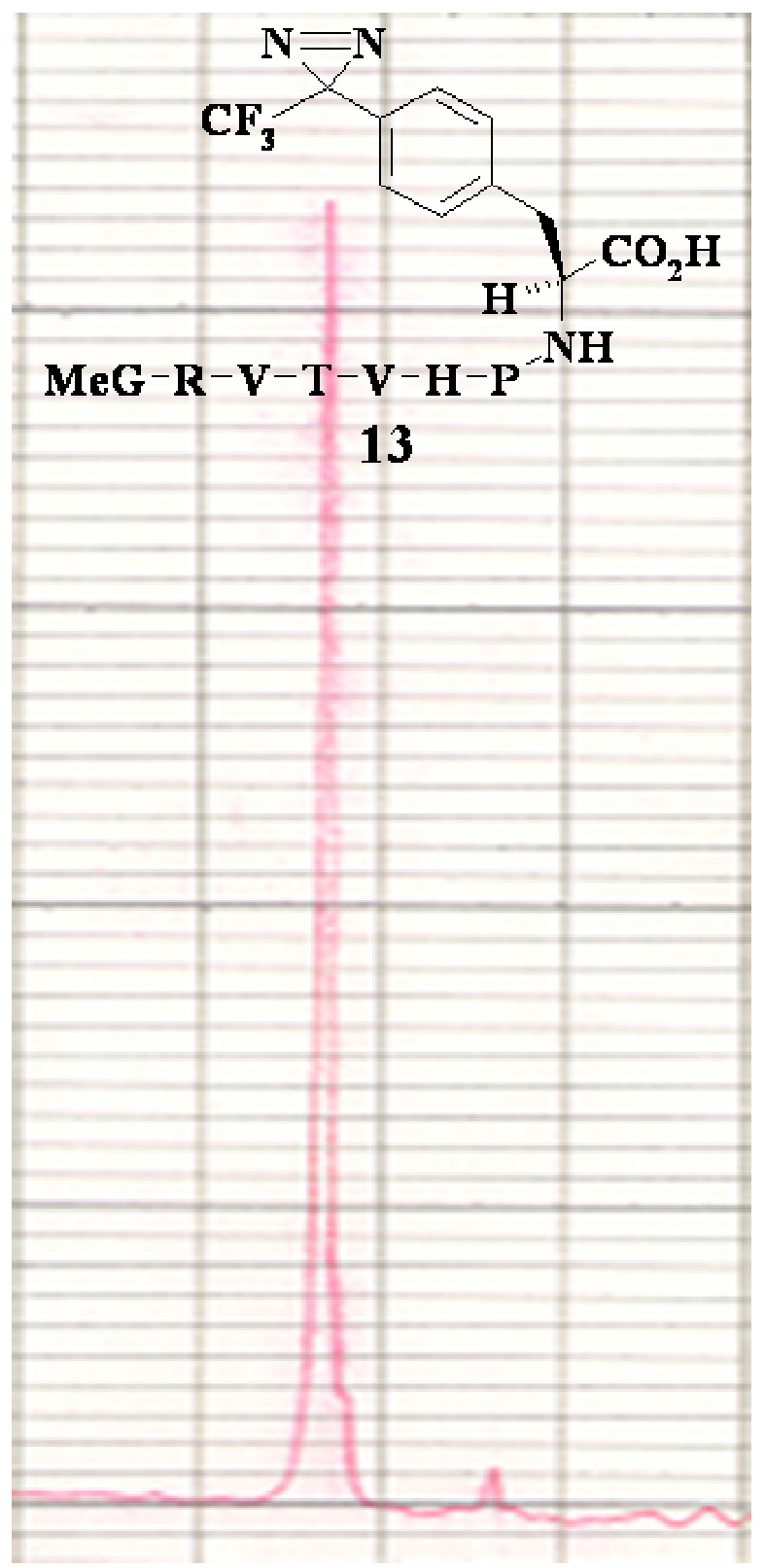


$\underline{\text { MALDI/TOF-MS spectra for compound } \mathbf{1 3}}$

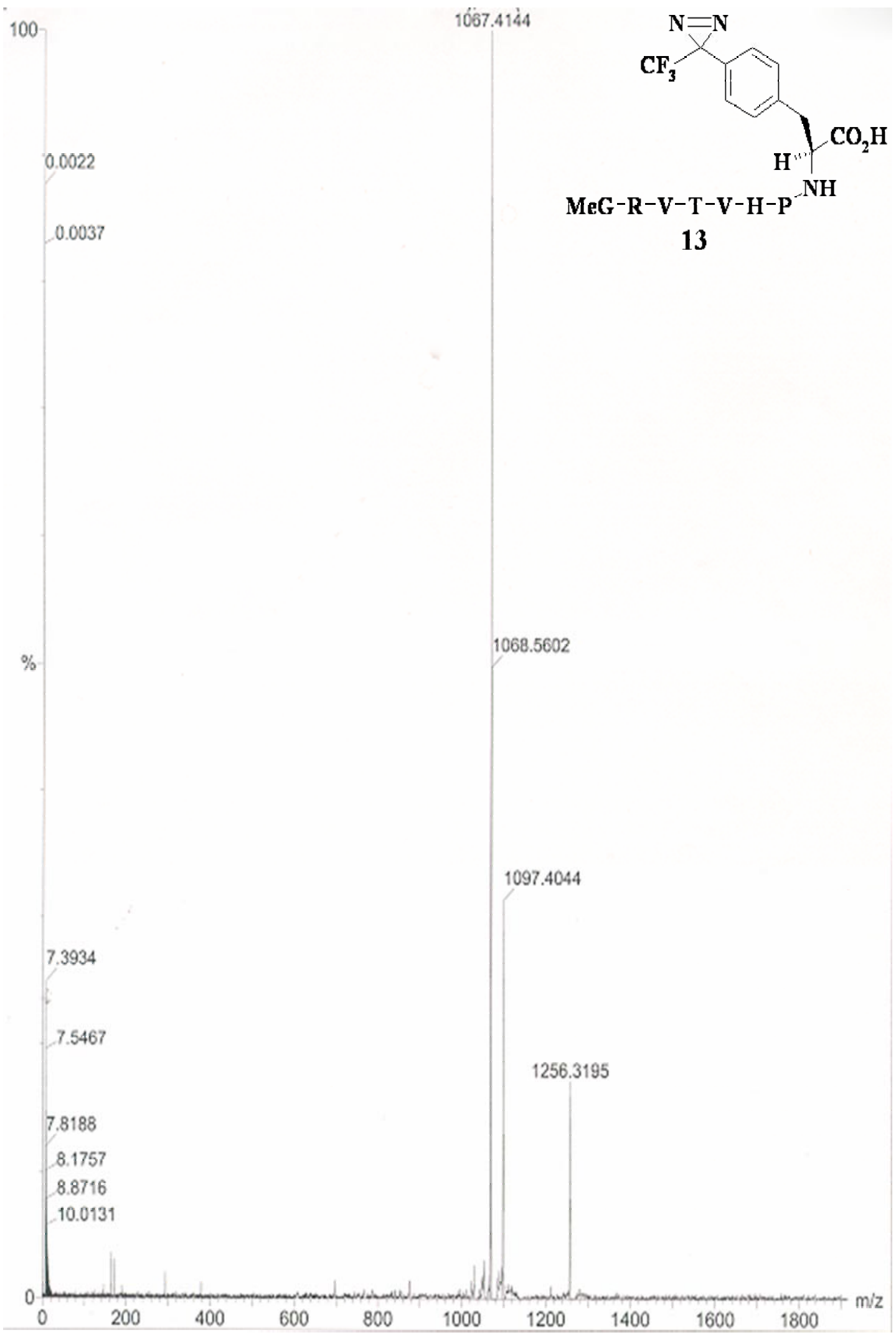

